\title{
Identifying Task Boundaries in Digital Assistants
}

\author{
Madian Khabsa ${ }^{1}$, Ahmed El Kholy ${ }^{2}$, Ahmed Hassan Awadallah ${ }^{2}$, Imed Zitouni ${ }^{2}$, Milad Shokouhi ${ }^{2 *}$ \\ Apple $\mathrm{Inc}^{1}$, Microsoft Corp ${ }^{2}$ \\ madian@apple.com,\{ahelkhol,hassanam,izitouni,milads\}@microsoft.com
}

\begin{abstract}
Digital assistants are emerging to become more prevalent in our daily lives. In interacting with these assistants, users may engage in multiple tasks within a short period of time. Identifying task boundaries and isolating them within a session is critical for measuring the performance of the system on each individual task. In this paper we aim to automatically identify sequences of interactions that together form a task. To this end, we sample interactions from a real world digital assistant and use crowd judges to segment a session into multiple tasks. After that, we use a machine learned model to identify task boundaries. Our learned model with its features significantly outperform the baselines. To the best of our knowledge, this is the first work that aims to identify tasks within digital assistant sessions.
\end{abstract}

\section{INTRODUCTION}

Digital assistants (DA) such as Siri and Cortana are emerging to be part of our daily lives, with the entire market of virtual assistants projected to grow $34 \%$ year over year ${ }^{1}$. These assistants are designed to accomplish multiple tasks. They could be goal-oriented tasks in which the user is trying to accomplish a certain goal, like setting a reminder. Or they could be tasks that seek information about a specific entity, for example getting the phone number of a nearby restaurant. Because assistants are designed to converse with users interactively, they can also perform some chitchatting tasks. From a user perspective, the fluidity of the assistant's responses encourage alternating between multiple types of tasks within a short period of time. For digital assistant developers, it is imperative to track the user interactions with the system and identify successful interactions from failed ones. Since a given session might contain multiple interleaving tasks, the assistant might have succeeded in accomplishing some of them, while failed at others. Therefore, it is crucial to segment user interaction with the assistant into multiple tasks, where the success of each task is independently measured.

While identifying task boundaries and measuring success in web search has been studied before [2], we are not aware of any work that tried to automatically segment digital assistant sessions into tasks. Therefore, we start by studying the access logs of a real world digital assistant, and analyzing what percentage of sessions do indeed contain more than one task. After that we use crowd workers to build a dataset of tasks within sessions, and train a machine

\footnotetext{
${ }^{*}$ Work done when all authors were at Microsoft

${ }^{1}$ https://www.gminsights.com/industry-analysis/intelligent-virtual-assistant-iva-market

This paper is published under the Creative Commons Attribution 4.0 International (CC BY 4.0) license. Authors reserve their rights to disseminate the work on their personal and corporate Web sites with the appropriate attribution.

WWW'18 Companion, April 23-27, 2018, Lyon, France

() 2018 IW3C2 (International World Wide Web Conference Committee), published

under Creative Commons CC BY 4.0 License.

ACM ISBN 978-1-4503-5640-4/18/04.

https://doi.org/10.1145/3184558.3186952
}

learning model to identify task boundaries. The problem is modeled as a binary classification between every pair of consecutive interactions. The classifier's job is to decide whether two consecutive interactions are part of the same task or not. A combination of features is used that is shown to outperform multiple baselines.

There are two main challenges that differentiate task identification in digital assistants from typical search engines. First, digital assistants comprise multiple sequential components like Automatic Speech Recognition (ASR) and Dialogue State Tracking (DST). Each of these components might fail independently and introduce errors to the user input (query). Whereas in search engines, the system receives the query exactly as it was typed. Second, because users interact with digital assistants in natural language, approaches based on syntactic features and edit distance are less effective.

\section{DATA}

The data used in this study comes from user access logs of a commercial digital assistant. We first started by studying the distribution of session length for the DA users. We collected all sessions for a period of 3 months and examined the number of interactions in the session against the frequency of such length. Figure 1a shows the distribution of session length as measured by the number of interactions. As seen in the figure, more than $40 \%$ of the sessions were found to contain 2 or more interactions. After that, we sampled 500 sessions from the logs of the same DA making sure each session contains 2 or more interactions. We use the standard session definition that is used in search engines wherein a period of inactivity of 30 minutes or more separates two sessions. Each sampled sessions was shown to 3 judges. The judges were asked to review the entire session, then flag the interaction that marks the end of the current task. By definition, the first interaction in a session starts a new task. For each interaction the judge viewed the user input, system response, along with the timestamps. Because the logs are stored in privacy preserving manner, certain inputs from the user and responses from the system are redacted.

In collecting the labels, a task was defined as follows: a sequence of requests are considered to be part of a single task if they collectively try to achieve a certain goal. There are two properties that determine if a request belongs to the current task or not: (1) Reformulation: The user is trying to repeat himself in a different way to achieve the same goal of a previous request. (2) Dependence: The current request depends on the output of the previous request. That is, the user depends on the DA response to issue the following request.

The judges generated 1631 data points, each data point corresponds to the label between two consecutive instances within the same session. Overall 929 instances indicated that the following interaction belongs to the same task as the previous one. Whereas, 703 instances corresponded to the following interaction being part of a separate task. The kappa inter-annotator agreement was 0.74 which indicates a substantial agreement among the judges. We further analyzed the tasks identified by the judges to understand how 


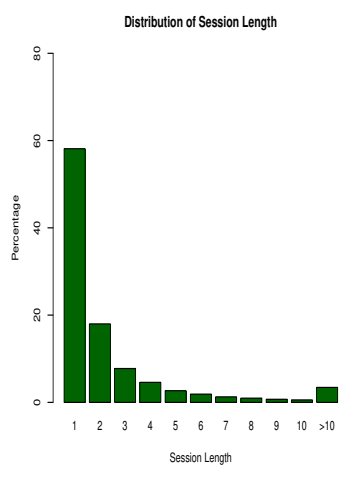

(a)

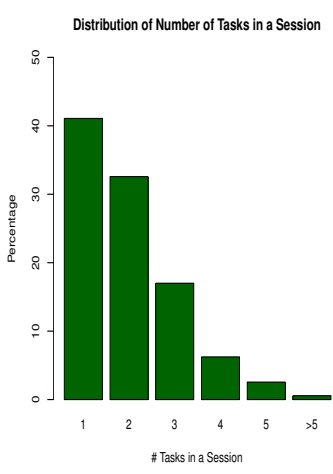

(b)
Figure 1: Figure (a) shows distribution of session length in terms of the number of interactions within the session. Figure (b) shows the distribution of number of tasks in sessions that contains 2 or more interactions

common is it for sessions to contain multiple tasks. The distribution of number of tasks in a session is plotted in Figure 1b. From the plot, we find that for sessions with multiple interactions, nearly $60 \%$ of them contain 2 or more tasks. This result motivates the need to develop an approach to automatically identify task boundaries.

Table 1: The performance of the task boundary detection classifier using different combination of features. + sign indicates the feature being added to all previous features. Baseline 1: a majority classifier, and Baseline 2 is [3]

\begin{tabular}{|l|l|l|l|l|l|l|}
\hline & \multicolumn{3}{|l|}{ Start of new task } & \multicolumn{3}{l|}{ Continue Same Task } \\
\hline Features & $\mathrm{P}$ & $\mathrm{R}$ & $\mathrm{F} 1$ & $\mathrm{P}$ & $\mathrm{R}$ & $\mathrm{F} 1$ \\
\hline Text & 0.62 & 0.61 & 0.62 & 0.71 & 0.71 & 0.71 \\
\hline + NER & 0.63 & 0.64 & 0.63 & 0.71 & 0.71 & 0.72 \\
\hline + Time & 0.70 & 0.67 & 0.68 & 0.76 & 0.78 & 0.77 \\
\hline + Embedding & 0.69 & 0.67 & 0.68 & 0.75 & 0.77 & 0.76 \\
\hline + Metaphone & 0.70 & 0.67 & 0.69 & 0.76 & 0.78 & 0.77 \\
\hline + Response & 0.70 & 0.66 & 0.68 & 0.76 & 0.78 & 0.77 \\
\hline + RequestNgram & $\mathbf{0 . 7 2}$ & 0.75 & 0.73 & 0.80 & $\mathbf{0 . 7 8}$ & $\mathbf{0 . 7 9}$ \\
\hline + ResponseNgram & 0.71 & $\mathbf{0 . 7 6}$ & $\mathbf{0 . 7 4}$ & $\mathbf{0 . 8 1}$ & 0.77 & 0.79 \\
\hline \hline Baseline 1 & 0 & 0 & 0 & 0.57 & 1 & 0.73 \\
\hline Baseline 2 & 0.68 & 0.26 & 0.38 & 0.62 & 0.9 & 0.74 \\
\hline
\end{tabular}

\section{APPROACH}

Similar to [1,2] we formulate the problem of identifying task boundaries as a binary classification task where the goal is to predict for every pair of consecutive user requests $r_{i}$ and $r_{i+1}$ if they belong to the same task or not. Random Forest is used as a classifier since it outperform other classifiers we experimented with. Model hyperparameters were tuned using grid search. The following features were used in the classifier: Text: A collection of features derived from textual similarity between the two consecutive requests. The features include Levenshtein distance, Jaccard similarity, longest common substring, shared prefix and postfix, and number of shared words.
NER: The number of shared named entities between the two pair of requests. Time: The time delta in seconds between the two consecutive requests Embedding: Word2Vec derived features between the two consecutive requests. This feature computes the number and ratio of words whose embedding vectors are close. Metaphone: The number and ratio of common metaphones between the words of the consecutive requests. This feature captures reformulations that are due to errors in speech recognition. Response: A collection of textual features that are derived from the two consecutive responses of the DA. They are similar to textual features described above. RequestNgram and ResponseNgram: Unigrams and Bigrams of the two consecutive requests and responses, respectively.

\section{RESULTS}

We use the dataset collected in Section 2 as the gold standard used for training and testing. The results of the classifier on a 5 fold cross validation are reported in Table 1 . In the table, the first row indicates using Text features only, whereas every subsequent row denotes the results of using the specified feature in addition to every feature listed above it in the table. For example, the row with feature + ResponseNgram indicates the performance when every listed feature was used in the classifier. From Table 1, we see that the classifier is able to identify more than $75 \%$ of the requests that mark the beginning of a new task, with $71 \%$ precision. We notice that the Time feature is responsible for a significant boost in the precision, whereas adding Ngram features lead to increase in recall. To get an understanding about how good or bad our classifier is, we compare the performance against the following 2 baselines. Baseline 1 is simple a majority class classifier that classifies every instance as Continue Same Task. In other words, every session contains one task only. Baseline 2 is based on the work of Mehrotra [3] wherein we will assume that a new session as defined by [3] corresponds to a new task. This baseline is basically a time delta classifier that was learned from a Mixture of Gaussians [3]. As seen in Table 1, our approach significantly outperforms both baselines. We also wanted to measure the performance of the task boundary segmentation classifier at the session level instead of the pairwise request level. In other words, given a session $S$ that contains $n$ request and response pairs that form $m$ tasks, we wanted to test the accuracy of the classifier in retrieving all $m$ tasks of the session. The classifier built using all the features achieved an accuracy of $46.8 \%$. Note that this is a hard task because it requires the classifier to make the correct prediction in each of the $n$ request/response pairs, and a single mistake results in an inaccurate session segmentation. This suggests that although our approach is able to perform generally well on identifying the start of new task, there is still room for improvement at accurately segmenting an entire session.

\section{REFERENCES}

[1] Rosie Jones and Kristina Lisa Klinkner. 2008. Beyond the session timeout: automatic hierarchical segmentation of search topics in query logs. In Proceedings of the 17th ACM conference on Information and knowledge management. ACM, 699-708.

[2] Claudio Lucchese, Salvatore Orlando, Raffaele Perego, Fabrizio Silvestri, and Gabriele Tolomei. 2011. Identifying task-based sessions in search engine query logs. In Proceedings of the fourth ACM international conference on Web search and data mining. ACM, 277-286.

[3] Rishabh Mehrotra, Ahmed El Kholy, Imez Zitouni, Milad Shokouhi, and Ahmed Hassan. 2017. Identifying User Sessions in Interactions with Intelligent Digital Assistants. In Proceedings of the 26th International Conference on World Wide Web Companion. 821-822. 